\title{
Construction of a Double Congenic Strain to Prove an Epistatic Interaction on Blood Pressure between Rat Chromosomes 2 and 10
}

\author{
John P. Rapp, Michael R. Garrett, and Alan Y. Deng \\ Department of Physiology and Molecular Medicine, Medical College of Ohio, Toledo, Ohio 43614-5804
}

\begin{abstract}
Previously we presented suggestive evidence from an $\mathrm{F}_{2}$ segregating population for an interaction on blood pressure (BP) between quantitative trait loci (QTL) on rat chromosomes (Chr) 2 and 10. To prove the existence of such an interaction, we developed congenic strains for $\mathrm{Chr} 2$ and 10 by introgressing the low BP QTL alleles into the Dahl salt-sensitive (S) strain. A double congenic strain was also constructed with both the Chr 2 and 10 low BP QTL alleles on the $\mathrm{S}$ background. The four strains ( $\mathrm{S}, \mathrm{Chr} 2$ congenic, $\mathrm{Chr}$ 10 congenic, and $\mathrm{Chr} 2 / 10$ double congenic) were studied for $B P$ response to increased salt intake. An analysis of variance showed significant main effects of $\mathrm{Chr} 2, \mathrm{Chr} 10$, and a significant interaction between $\mathrm{Chr} 2$ and 10 on $\mathrm{BP}$ and heart weight (all $P<0.0001$ ). The interaction accounted for $24 \mathrm{mmHg}$ of BP and $79 \mathrm{mg}$ of heart weight. Thus, the discovery and proof of epistatic interactions are clearly critical to understanding the genetics of blood pressure. (J. Clin. Invest. 1998. 101:1591-1595.) Key words: hypertension • Dahl rats $\bullet$ salt sensitivity $\bullet$ quantitative trait loci $\bullet$ heart weight
\end{abstract}

\section{Introduction}

In the genetic analysis of polygenic traits such as blood pressure (BP), genetic markers are sought that show genetic linkage to the trait. Analysis of such data allows only a statistical estimate of the existence and location of the loci involved, socalled quantitative trait loci $(\mathrm{QTL})^{1}(1,2)$. As a first approximation, the effects of QTL are assumed to be additive, although it is obvious that interactions between or among loci (epistatic interactions) are possible, if not likely $(2,3)$.

QTL linkage analysis in experimental segregating populations is often followed by the construction of congenic strains to either prove or refute the existence of a QTL $(2,4,5)$. In this technique a chromosomal region containing a putative QTL is introgressed from one strain (donor) into another (re-

Address correspondence to John P. Rapp, Department of Physiology and Molecular Medicine, Medical College of Ohio, Block Health Science Building, 3035 Arlington Avenue, Toledo, Ohio 43614-5804. Phone: 419-383-4297. FAX: 419-383-6168.

Received for publication 12 November 1997 and accepted in revised form 26 November 1998.

1. Abbreviations used in this paper: $\mathrm{BW}$, body weight; HW, heart weight; MNS, Milan normotensive strain; QTL, quantitative trait locus; S, inbred salt-sensitive rats; WKY, Wistar Kyoto rats.

J. Clin. Invest.

(C) The American Society for Clinical Investigation, Inc. 0021-9738/98/04/1591/05 \$2.00

Volume 101, Number 8, April 1998, 1591-1595

http://www.jci.org cipient) by a breeding protocol involving repeated back-crossing to the recipient strain with selection for the donor chromosomal region (5-7). In our experiments we are always placing a low BP QTL allele from a normotensive rat strain onto the genetic background of a hypertensive strain, i.e., the inbred Dahl salt-sensitive (S) rat. The existence of the QTL is proven if the resulting congenic strain has a lower $\mathrm{BP}$ than the $\mathrm{S}$ strain. This general approach is also being applied to other polygenic disease traits such as diabetes mellitus (8-10), epilepsy (11), tumor susceptibility $(12,13)$, and systemic lupus erythematosis (14).

In a previous linkage analysis of an $\mathrm{F}_{2}$ population, we observed statistical evidence for BP QTL on rat chromosomes (Chr) 2 and 10, and weak statistical evidence for an interaction between these QTL on BP (15). Subsequently, separate congenic strains were constructed on the $\mathrm{S}$ background for the appropriate regions of $\mathrm{Chr} 2$ or 10 donated by a normotensive strain $(16,17)$. Each congenic strain had markedly lower BP than the S rats, proving the existence of QTL in the chromosomal segments transferred. In the present work, a double congenic strain was constructed containing the low BP QTL alleles from both Chr 2 and 10 on the S genetic background. Analysis of the BP of this double congenic strain, the two single congenic strains, and the $\mathrm{S}$ strain provides definitive evidence for a surprisingly strong epistatic interaction on BP of the QTL on Chr 2 and 10. To our knowledge, this is the only such analysis for an interaction on a quantitative trait in a mammalian system. In recent studies of tumor susceptibility in mice, a related technique of crossing two recombinant congenic strains also provided strong evidence for QTL interactions $(12,13)$.

\section{Methods}

Rat strains. Inbred Dahl salt-sensitive (SS/Jr) rats were from our colony at the Medical College of Ohio; these rats will be referred to hereafter as $\mathrm{S}$ rats. Two congenic strains previously developed by us were also used. In both cases, a segment of chromosome containing a low BP QTL allele had been introgressed into the S strain. The donor strain for Chr 2 congenic strain was Wistar Kyoto (WKY, Harlan Sprague Dawley, Indianapolis, IN) and the Chr 2 segment introgressed is shown in Fig. 1. The strain was originally named S.WKYD2N35/Nep (16), but will be referred to here as the Chr 2 congenic. The donor strain for the $\mathrm{Chr} 10$ congenic strain was the Milan normotensive strain (MNS) obtained from the Genetic Resource Section of the National Institutes of Health (Bethesda, MD), and the Chr 10 segment introgressed is also shown in Fig. 1. The strain was originally named S.M(10b) (17), but will be referred to here as the Chr 10 congenic.

We constructed a double congenic, i.e., a single strain in which both the Chr 2 and 10 regions were on the $\mathrm{S}$ genetic background. The double congenic will be called the Chr 2/10 double congenic; it was constructed as follows. In this presentation $W$ refers to the WKY allele, $M$ the MNS allele, and $S$ the S-rat allele. The Chr 2 and 10 congenic strains were crossed and this $\mathrm{F}_{1}$ was back-crossed to the $\mathrm{Chr} 2$ congenic. Rats that were (a) homozygous $W W$ at all loci in the Chr 2 congenic segment of chromosome and (b) $M S$ in the Chr 10 congenic 


\section{CHR 2}

CHR 2 CONGENIC

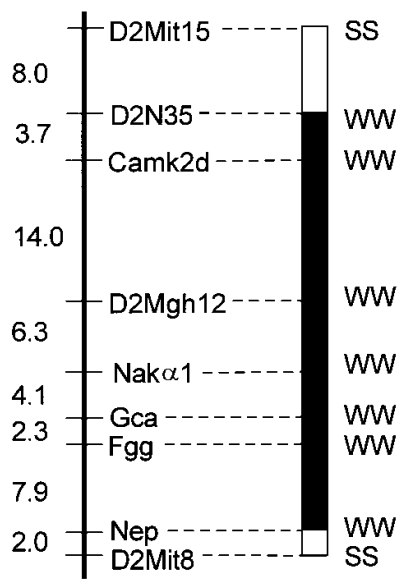

CHR 10

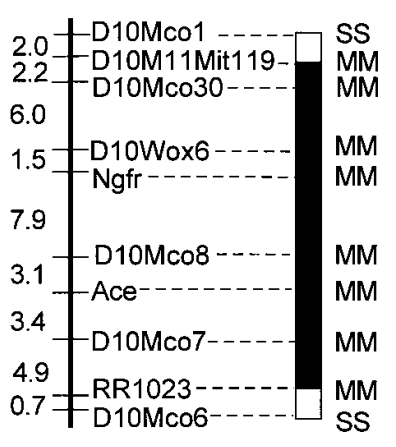

Figure 1. Genetic maps of the regions used in the construction of congenic strains. Genetic distances are in centiMorgans with Kosambi's correction. The black bar to the right of each map represents the segment introgressed into the $\mathrm{S}$ strain; and the open bars on the ends of these segments mark the region in which recombination occurred. The Chr 2 segment was from the WKY strain (donating alleles designated $W$ ) and the Chr 10 segment was from the MNS strain (donating alleles designated $M$ ). $S$ denote alleles from $\mathrm{S}$ rats.

segment were selected and crossed with each other. Offspring that were $M M$ at the Chr 10 congenic segment (and of course all were $W W$ at the Chr 2 segment) were selected and bred to produce the Chr $2 / 10$ double congenic strain. The double congenic was maintained by brother-sister mating for one generation, and then three litters were genotyped at all relevant loci on Chr 2 and 10 to be sure these regions were homozygous for the appropriate alleles. The strain was then maintained by brother-sister mating.

Genotyping. DNA for genotyping was obtained from tail biopsy tissue extracted with the QIAamp Tissue Kit (Qiagen Inc., Chatsworth, CA). All genetic markers were based on amplification by the polymerase chain reaction of polymorphic microsatellites. Oligonucleotide primer pairs for genetic markers and details of the techniques were given previously (15-17).

$B P$. S, Chr 2 congenic, Chr 10 congenic, and $\mathrm{Chr} 2 / 10$ double congenic rats were bred concomitantly. Litters were culled to 8-10 rats/ litter 3-4 d after birth, weaned at $30 \mathrm{~d}$ of age, and fed Teklad diet 3074 (Harlan Teklad, Madison, WI) containing 0.2\% NaCl. 12 male rats of each strain born within $3 \mathrm{~d}$ of each other were used from at least four different litters of each strain. Rats were housed four to a cage, one rat from each strain. Rats were identified with a numbered skin clip placed in the back of the neck (National Band and Tag Co., Newport, KY). When the rats were 45-48-d old, they were fed Teklad diet 94217 containing $2 \% \mathrm{NaCl}$. After $24 \mathrm{~d}$ on $2 \% \mathrm{NaCl}$ diet, $\mathrm{BP}$ was taken at daily sessions for four consecutive days by the tail cuff method (18) in the conscious, restrained rat with equipment made by IITC, Inc. (Woodland Hills, CA). Rats were warmed to $28^{\circ} \mathrm{C}$ for BP measurement. At least three consistent $\mathrm{BP}$ readings at a given daily session were obtained and averaged as that session's reading. The four sessions for each rat were performed by two different operators, who did not know the identity of the rats; each operator performed two sessions on each rat. The averages of all four sessions' readings were averaged as the BP of the rat. Rats were killed with $\mathrm{CO}_{2}$, and body and heart weights were measured.

Two separate experiments (blocks) were performed to evaluate BP. Each block contained 12 rats/strain (i.e., 48 rats per block); when the two blocks were combined, data were available from 24 rats/strain (or a total of 96 rats).
Statistical analysis. Programs used in statistical analysis were from SPSS (Chicago, IL). The experimental design was analyzed as a $2 \times 2$ factorial ANOVA with two replicate blocks. The main effects were (a) genotype of the congenic region on Chr 10 (either $S S$ or $M M)$ or $(b)$ genotype of the congenic region on Chr 2 (either $S S$ or $W W$ ). The $2 \times 2$ ANOVA also provided an evaluation of the interaction between the genotypes on Chr 2 and 10. The data were also analyzed as a one-way ANOVA followed by contrasts among the strains using the Scheffé procedure (19).

\section{Results}

$B P$. There was no difference between the replicate blocks for BP by ANOVA. Thus, the blocks were combined and the combined data will be presented in detail. It is noted in passing, however, that for each block alone there were significant effects on BP of Chr 2 (both blocks $P<0.0001$ ), Chr 10 (both blocks $P<0.0001)$, and a significant interaction between $\mathrm{Chr} 2$ and $10(P=0.006$ block $1, P<0.0001$ block 2$)$ by a $2 \times 2$ factorial ANOVA. Of course, the data of the combined blocks were also highly significant for main effects and interaction (all $P<0.0001)$.

Although the $2 \times 2$ factorial ANOVA immediately reveals a statistical interaction between Chr 2 and 10 on BP, intuitive interpretation of such an interaction is a little obtuse. A more meaningful presentation is given in Fig. 2. It is obvious by inspection of Fig. 2 that all three congenics have markedly reduced BP compared to $\mathrm{S}$ as expected (Scheffé contrasts $P<$ 0.0001 following a one-way ANOVA). In presenting the data for further interpretation, however, we have taken the BP of the Chr 2/10 double congenic as a baseline and considered increases in BP in the other strains from that "control" level. The double congenic is, of course, essentially an S rat, except that for the QTL on Chr 2 and 10 it has low BP alleles ( $W W$ on Chr 2 and $M M$ on Chr 10) substituted for the high BP SS genotype. Because all the proven $\mathrm{QTL}^{2}$ on chromosomes other than 2 and 10 contain high BP alleles in the double congenic, it is not surprising that the double congenic has a relatively high (systolic) $\mathrm{BP}$ of $170 \pm 1.4 \mathrm{mmHg}$ (mean $\pm \mathrm{SE}$ ) on $2 \% \mathrm{NaCl}$ diet.

Changing the BP QTL on Chr 2 in the double congenic from the low $W W$ genotype to the high $S S$ genotype is represented by the Chr 10 congenic (Fig. 2). Such substitution increased BP from $170 \pm 1.4$ to $178 \pm 1.9 \mathrm{mmHg}$ or $8 \mathrm{mmHg}$, labeled as increment $a$ in Fig. 2. Changing the BP QTL on Chr 10 in the double congenic from the low $M M$ genotype to the high $S S$ genotype is represented by the Chr 2 congenic (Fig. 2). Such substitution increased BP from $170 \pm 1.4$ to $185 \pm 1.8$ $\mathrm{mmHg}$ or $15 \mathrm{mmHg}$, labeled increment $b$ in Fig. 2. On the assumption that the QTL alleles on Chr 2 and 10 were additive, the expected increase in BP upon changing from low to high $\mathrm{BP}$ alleles on both $\mathrm{Chr} 2$ and 10 would be the increment labeled $a+b$ in Fig. 2 , or $23 \mathrm{mmHg}$. The actual effect is to increase BP from $170 \pm 1.4$ to $217 \pm 3.4 \mathrm{mmHg}$ in the S strain, an increase of $47 \mathrm{mmHg}$. The increment above the $23 \mathrm{mmHg}$ expected, i.e., $47-23$ or $24 \mathrm{mmHg}$, represents the component due

2. The QTL detected using Dahl S rats other than those on Chr 2 and 10 are on $\operatorname{Chr} 1,3,5,7,9$, and 13 , all of which have been proven to exist by the construction on congenic strains, and all of which have the high BP allele in the S strain. The data for Chr 13 (20) and 7 (21) are published and the remainder are in preparation (Rapp, J.P., M. Garrett, and G.T. Cicila). 


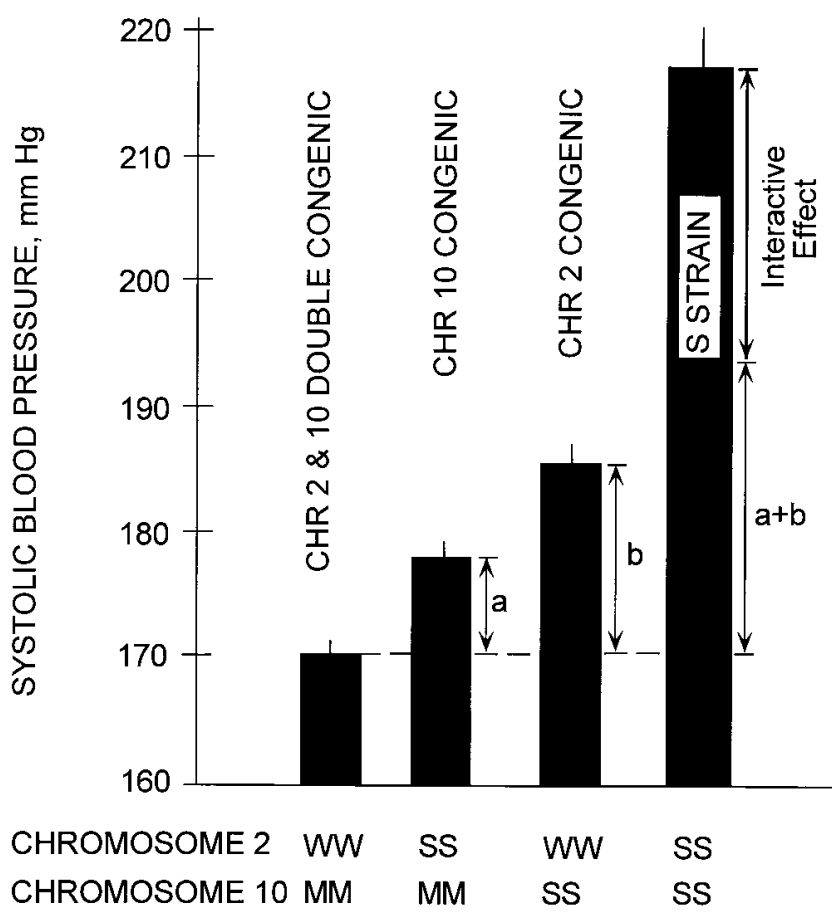

Figure 2. Bar graph for systolic BP of $\mathrm{S}$ and the congenic strains. Genotypes in the congenic regions shown in Fig. 1 for Chr 2 and 10 are given at the bottom of the figure. The increments labeled $a, b$, and $a+b$ assume additive effects on $\mathrm{BP}$, and the increment labeled interactive effect results from the interaction of the QTL on Chr 2 and 10 (see text). very similar results for adjusted $\mathrm{HW}$ and BP. First, it is obvious (and ANOVA confirmed, all $P<0.0001$ ) that substituting the QTL region of Chr 2 or 10 into $S$ rats reduced adjusted HW. The increments of adjusted HW defined in Fig. 3 were $a=12$ $\mathrm{mg}, b=60 \mathrm{mg},(a+b)=72 \mathrm{mg}$, and the interactive effect was $79 \mathrm{mg}$, which was highly significant $(P<0.0001)$.

Cursory examination of Figs. 2 and 3 indicates that adjusted HW and BP must be correlated. This was the case $(r=$ $0.71, P<0.0001$ using all 96 rats). The regression of adjusted $\mathrm{HW}$ on $\mathrm{BP}$ was used to remove the effect of BP on HW. A $2 \times 2$ factorial ANOVA of HW (now adjusted for BW, blocks, and BP) showed that the effect of Chr 2 on $\mathrm{HW}$ was removed $(P=$ 0.91), a small effect of Chr 10 still remained (genotype $S S$ on Chr 10 was $26 \mathrm{mg}$ heavier than genotype $M M, P=0.022)$, and there was no interaction between $\mathrm{Chr} 2$ and $10(P=0.46)$.

\section{Discussion}

Originally (15) we described an interaction between Chr 2 and 10 on $\mathrm{BP}$ in an $\mathrm{F}_{2}$ population derived from a cross of $\mathrm{S}$ with the MNS. Thus, in that experiment, we were comparing $\mathrm{S}$ versus MNS QTL alleles on Chr 2 and 10. In the present double congenic, the Chr 2 QTL allele was donated by the WKY strain. We used the Chr 2 congenic with the WKY QTL allele, rather than the Chr 2 congenic with the MNS QTL allele, because the former has a smaller introgressed chromosomal segment than the latter. Also, we wanted to determine if the interaction was dependent on the presence of only the MNS Chr 2 allele, which is obviously not the case.

There are guidelines in the literature for what constitutes statistical significance in a genome scan (to detect additive QTL; 24). There are, however, no such guidelines for statistical to the interaction between $\mathrm{Chr} 2$ and 10 on the $\mathrm{S}$ genetic background (labeled interactive effect in Fig. 2). This $24 \mathrm{mmHg}$ interactive component is tested formally by a one-way ANOVA on BP (which showed significant $P<0.0001$ differences among strains) followed by the contrast ( $\mathrm{S}+\mathrm{Chr} 2 / 10$ double congenic) - (Chr 2 congenic + Chr 10 congenic), which should equal zero under the null hypothesis of no interaction. This null hypothesis is rejected by Scheffé's test at $P<0.0001$, thus the interaction is significant. This is equivalent to the interaction term from a $2 \times 2$ factorial ANOVA (22). The interaction was still significant $(P<0.0001)$ when the data were transformed by taking the logarithm, square root, or reciprocal of BP.

Heart weight $(H W)$. Analysis of HW was confounded by the fact that the factorial ANOVA showed that body weight (BW) was: $(a)$ increased $19 \mathrm{~g}$ by the Chr 2 substitution in $\mathrm{S}$ rats $(P<0.0001)$; $(b)$ decreased by $7 \mathrm{~g}$ by the Chr 10 substitution in $\mathrm{S}$ rats $(P=0.019)$; and $(c)$ the blocks differed by $10 \mathrm{~g}(P=$ $0.001)$. The overall population mean BW was $330 \mathrm{~g}$. Because there was a correlation ( $r=0.51, P<0.0001$ using all 96 rats $)$ between HW and BW a regression equation was fitted to the data and HW was adjusted for differences in BW to yield adjusted HW (23). Because adjusted HW still showed a difference between blocks, a correction was made for this difference as well. Data on the HW adjusted for these variants are presented in Fig. 3.

The analysis-adjusted HW in Fig. 3 proceeded as detailed above for BP in Fig. 2. Comparison of Figs. 2 and 3 indicates

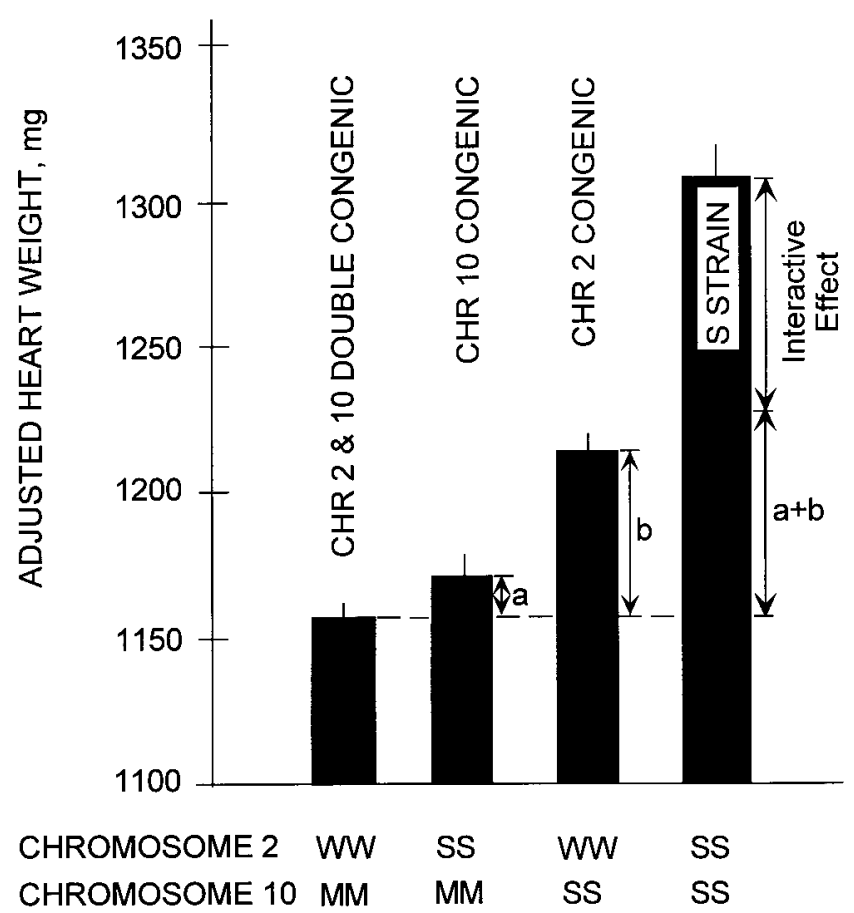

Figure 3. Bar graph for HW adjusted for differences in BW of S and congenic strains. The format of the figure is identical to that of Fig. 2. 
detection of interactions that present special problems of detection $(2,3)$. Our original description of the Chr 2 and 10 interaction in an $\mathrm{F}_{2}$ population with a $P=0.061$ would certainly be marginal under any criteria. Nevertheless the double congenic proves beyond reasonable doubt that this interaction is real. Thus, one is left with the realization that sole reliance on statistical guidelines would result in a failure to find some potentially important effects. Such a caveat is, of course, also recognized by statisticians $(3,24-27)$.

The genetic interaction between Chr 2 and 10 on BP certainly means that the genes involved code for molecules involved in BP regulatory pathways that interact at some level. With this in mind, the discovery of one or the other of the interacting QTL might aid in uncovering the remaining QTL. In the most useful case, one QTL might code for a protein that binds directly to a protein coded by the other QTL.

In our work, we consider HW to reflect relatively large chronic changes in $\mathrm{BP}$, as the heart hypertrophies working against increased BP. Thus, the strain changes in BP and HW observed here are interpreted to result from the same underlying genetic causes. That is not to deny that HW may be influenced by genetic factors independent of BP (28-32), but such phenomena are not a major component of the work presented here. There was only weak evidence for an effect of Chr 10 on HW independent of BP. In another study (32) involving an $\mathrm{F}_{2}$ cross-derived from genetically hypertensive and brown Norway rats, an interaction between Chr 2 and 10 was observed for $\mathrm{HW}$ but not for BP.

The QTL regions shown in Fig. 1 used in the construction of the double congenic strain contain some candidate genes that could mediate BP effects. On Chr 2 neutral endopeptidase $(N e p)$, which degrades atrial natriuretic peptide, guanylyl cyclase A $(G c a)$, which is one of the receptors for atrial natriuretic peptide, and NaK-ATPase $\alpha 1$ isoform, which is important in $\mathrm{Na}^{+}$and $\mathrm{K}^{+}$transport in kidney and vascular smooth muscle cells, can all be construed as candidate genes. Calmodulin kinase $(C a m k 2 d)$ might also be considered a candidate gene, but that region of Chr 2 has subsequently been shown not to contain the BP QTL by study of congenic substrains derived from the Chr 2 congenic strain (Rapp, J.P., and A.Y. Deng, unpublished observations). On Chr 10, angiotensin converting enzyme (Ace) is an obvious candidate, and nerve growth factor receptor $(N g f r)$, which mediates sympathetic nerve growth, has also been considered by some (33) to be a candidate gene. Among these candidates there are no obvious pairs that might explain the interaction observed between $\mathrm{Chr}$ 2 and 10 .

Recently it has been reported (34) by affected sib pair analysis that a region of human $\mathrm{Chr} 17$, homologous to the region of rat Chr 10 around the Ace locus, was linked to BP. Obviously, human studies should also look for linkage of BP to the human chromosomal region homologous to our rat $\mathrm{Chr} 2$ congenic region, and in addition, human studies should look for an interaction between the human chromosomal regions homologous to our rat $\mathrm{Chr} 2$ and 10 congenic segments.

\section{Acknowledgments}

This work was supported by grants HL20176 and HL55006 to J.P. Rapp from the National Institutes of Health and by the Helen and Harold McMaster Endowed Chair in Biochemistry and Molecular Bi- ology, and by grants 96006640 and NW-96-05-B to A.Y. Deng from the American Heart Association (National Center and Ohio affiliate, respectively).

\section{References}

1. Lander, E.S., and D. Botstein. 1989. Mapping Mendelian factors underlying quantitative traits using RFLP linkage maps. Genetics. 121:185-199.

2. Tanksley, S.D. 1993. Mapping polygenes. Annu. Rev. Genet. 27:205-233.

3. Frankel, W.N., and N.J. Schork. 1996. Who's afraid of epistasis? Nat. Genet. 14:371-373.

4. Lander, E.S., and N.J. Schork. 1994. Genetic dissection of complex traits. Science. 265:2037-2048.

5. Rapp, J.P., and A.Y. Deng. 1995. Detection and positional cloning of blood pressure quantitative trait loci: is it possible? Hypertension. 25:11211128.

6. Snell, G.D. 1948. Methods for the study of histocompatibility genes. $J$. Genetics. 49:87-108.

7. Silver, L.M. 1995. Mouse Genetics, Concepts and Applications. Oxford University Press, New York. 44-52.

8. Serreze, D.V., M. Prochazka, P.C. Reifsnyder, M.M. Bridgett, and E.H. Leiter. 1994. Use of recombinant congenic and congenic strains of NOD mice to identify a new insulin-dependent diabetes resistance gene. J. Exp. Med. 180: 1553-1558.

9. Lord, C.J., S.K. Bohlander, E.A. Hopes, C.T. Montague, N.J. Hill, J-B. Prins, R.J. Renjilian, L.B. Peterson, L.S. Wicker, J.A. Todd, and P. Denny. 1995. Mapping the diabetes polygene Idd3 on mouse chromosome 3 by use of novel congenic strains. Mamm. Genome. 6:563-570.

10. Yui, M.A., K. Muralidharan, B. Moreno-Altamirano, G. Perrin, K. Chestnut, and E.K. Wakeland. 1996. Production of congenic mouse strains carrying NOD-derived diabetogenic genetic intervals: an approach for the genetic dissection of complex traits. Mamm. Genome. 7:331-334.

11. Frankel, W.N., E.W. Johnson, and C.M. Lutz. 1995. Congenic strains reveal effects of the epilepsy quantitative trait locus, $E l 2$, separate from other $E l$ loci. Mamm. Genome. 6:839-843.

12. Fijneman, R.J.A., S.S. de Vries, R.C. Jansen, and P. Demant. 1996. Complex interactions of new quantitative trait loci, Sluc1, Sluc2, Sluc3, and Sluc4, that influence the susceptibility to lung cancer in the mouse. Nat. Genet. 14:465-467.

13. van Wezel, T., A.P.M. Stassen, C.J.A. Moen, A.A.M. Hart, M.A. van der Valk, and P. Demant. 1996. Gene interaction and single gene effects in colon tumour susceptibility in mice. Nat. Genet. 14:468-470.

14. Morel, L., Y. Yu, K.R. Blenman, R.A. Caldwell, and E.K. Wakeland 1996. Production of congenic mouse strains carrying genomic intervals containing SLE-susceptibility genes derived from the SLE-prone NZM2410 strain. Mamm. Genome. 7:335-339.

15. Deng, Y., and J.P. Rapp. 1992. Cosegregation of blood pressure with angiotensin converting enzyme and atrial natriuretic peptide receptor genes using Dahl salt-sensitive rats. Nat. Genet. 1:267-272.

16. Deng, A.Y., H. Dene, and J.P. Rapp. 1997. Congenic strains for the blood pressure quantitative trait locus on rat chromosome 2. Hypertension. 30: 199-202.

17. Dukhanina, O.I., H. Dene, A.Y. Deng, C.R. Choi, B. Hoebee, and J.P. Rapp. 1997. Linkage map and congenic strains to localize blood pressure QTL on rat chromosome 10. Mamm. Genome. 8:229-235.

18. Buñag, R.D., and J. Butterfield. 1982. Tail-cuff blood pressure measurement without external preheating in awake rats. Hypertension. 4:898-903.

19. Scheffé, H. 1959. The Analysis of Variance. John Wiley \& Sons, Inc., New York. 66-72.

20. Zhang, Q.Y., H. Dene, A.Y. Deng, M.R. Garrett, H.J. Jacob, and J.P. Rapp. 1997. Interval mapping and congenic strains for a blood pressure QTL on rat chromosome 13. Mamm. Genome. 8:636-641.

21. Cicila, G.T., O.I. Dukhanina, T.W. Kurtz, R. Walder, M.R. Garrett, H. Dene, and J.P. Rapp. 1997. Blood pressure and survival of a chromosome 7 congenic strain bred from Dahl rats. Mamm. Genome. 8:896-902.

22. Sokal, R.R., and F.J. Rohlf. 1969. Biometry. W.M. Freeman and Company, San Francisco. 309 pp

23. Ostle, B. 1963. Statistics in Research. Iowa State University Press, Ames, IA. 199-200.

24. Lander, E., and L. Kruglyak. 1995. Genetic dissection of complex traits: guidelines for interpreting and reporting linkage results. Nat. Genet. 11:241-247.

25. Witte, J.S., R.C. Elston, and N.J. Schork. 1996. Genetic dissection of complex traits (Correspondence). Nat. Genet. 12:355-356.

26. Curtis, D. 1996. Genetic dissection of complex traits (Correspondence). Nat. Genet. 12:356-357.

27. Lander, E., and L. Kruglyak. 1996. Genetic dissection of complex traits (Correspondence). Nat. Genet. 12:357-358.

28. Kunes, J., V. Kren, P. Klír, J. Zicha, and M. Pravenec. 1990. Genetic determination of heart and kidney weights studied using a set of recombinant inbred strains: the relationship to blood pressure. J. Hypertens. 8:1091-1095. 
29. Tanase, H., Y. Yamori, K.T. Hansen, and W. Lovenberg. 1982. Heart size in inbred strains of rats. Part 1. Genetic determination of the development of cardiovascular enlargement in rats. Hypertension. 4:864-872.

30. Pravenec, M., D. Gauguier, J-J. Schott, J. Buard, V. Kren, C. Bila, C. Szpirer, J. Szpirer, J-M. Wang, H. Huang, et al. 1995. Mapping of quantitative trait loci for blood pressure and cardiac mass in the rat by genome scanning of recombinant inbred strains. J. Clin. Invest. 96:1973-1978.

31. Hamet, P., M.A. Kaiser, Y. Sun, V. Page, M. Vincent, V. Kren, M. Pravenec, J. Kunes, J. Trembley, and N.J. Samani. 1996. HSP27 locus cosegregates with left ventricular mass independently of blood pressure. Hypertension. 28:1112-1117.

32. Harris, E.L., E.L. Phelan, C.M. Thompson, J.A. Millar, and M.R.
Grigor. 1995. Heart mass and blood pressure have separate genetic determinants in the New Zealand genetically hypertensive (GH) rat. J. Hypertens. 13: 397-404.

33. Kapuscinski, M., F. Charchar, B. Innes, G.A. Mitchell, T.L. Norman, and S.B. Harrap. 1996. Nerve growth factor gene and hypertension in spontaneously hypertensive rats. J. Hypertens. 14:191-197.

34. Julier, C., M. Delepine, B. Keavney, J. Terwilliger, S. Davis, D.E. Weeks, T. Bui, X. Jeunemaitre, G. Velho, P. Froguel, et al. 1997. Genetic susceptibility for human familial essential hypertension in a region of homology with blood pressure linkage on rat chromosome 10. Hum. Mol. Genet. 6:20772086. 